PROCEEDINGS OF THE

AMERICAN MATHEMATICAL SOCIETY

Volume 138, Number 7, July 2010, Pages 2381-2383

S 0002-9939(10)10336-0

Article electronically published on March 11, 2010

\title{
A NON-FINITELY GENERATED ALGEBRA OF FROBENIUS MAPS
}

\author{
MORDECHAI KATZMAN
}

(Communicated by Bernd Ulrich)

\begin{abstract}
This paper describes an Artinian module over a ring of prime characteristic whose algebra of Frobenius maps is not finitely generated. This settles a question raised by Lyubeznik and Smith.
\end{abstract}

\section{INTRODUCTION}

The purpose of this paper is to answer a question raised by Gennady Lyubeznik and Karen Smith in [LS]. This question involves the finite generation of a certain non-commutative algebra which we define below (cf. section 3 in [LS]).

Let $S$ be any commutative algebra of prime characteristic $p$. For any $S$-module $M$ and all $e \geq 0$ we let $\mathcal{F}^{e}(M)$ denote the set of all additive functions $\phi: M \rightarrow M$ with the property that $\phi(s m)=s^{p^{e}} \phi(m)$ for all $s \in S$ and $m \in M$. Note that for all $e_{1}, e_{2} \geq 0$ and $\phi_{1} \in \mathcal{F}^{e_{1}}(M), \phi_{2} \in \mathcal{F}^{e_{2}}(M)$, the composition $\phi_{2} \circ \phi_{1}$ is in $\mathcal{F}^{e_{1}+e_{2}}(M)$. Note also that each $\mathcal{F}^{e}(M)$ is a module over $\mathcal{F}^{0}(M)=\operatorname{Hom}_{S}(M, M)$ via $\phi_{0} \phi=\phi_{0} \circ \phi$. We now define $\mathcal{F}(M)=\bigoplus_{e>0} \mathcal{F}^{e}(M)$ and endow it with the structure of a $\operatorname{Hom}_{S}(M, M)$-algebra with multiplication given by composition.

In section 2 we construct an example of an Artinian module over a complete local ring $S$ for which $\mathcal{F}(M)$ is not a finitely generated $\operatorname{Hom}_{S}(M, M)$-algebra, thus giving a negative answer to the question raised in section 3 of [LS].

\section{The EXAMPLe}

Let $\mathbb{K}$ be a field of characteristic $p>0, R=\mathbb{K} \llbracket x, y, z \rrbracket$, and let $I \subseteq R$ be an ideal. Let $E$ be the injective hull of the residue field of $R$ and let $f$ denote the standard Frobenius map of $E$ (cf. section 4 in $[\mathrm{K}]$ ). Write $S=R / I$ and let $E_{S}$ be the injective hull of the residue field of $S$.

Notice that as $S$ is complete, $\mathcal{F}^{0}\left(E_{S}\right)=\operatorname{Hom}_{S}\left(E_{S}, E_{S}\right) \cong S$; the $S$-module $\mathcal{F}^{e}\left(E_{S}\right)$ of $p^{e}$ th Frobenius maps on $E_{S}$ is given by $\left(I^{\left[p^{e}\right]}: I\right) f^{e}$ (cf. section 4 in $[\mathrm{K}]$ ).

For all $e \geq 1$ write $K_{e}=\left(I^{\left[p^{e}\right]}: I\right)$. We define

$$
L_{e}=\sum_{\substack{1 \leq \beta_{1}, \ldots, \beta_{s}<e \\ \beta_{1}+\cdots+\beta_{s}=e}} K_{\beta_{1}} K_{\beta_{2}}^{\left[p^{\beta_{1}}\right]} K_{\beta_{3}}^{\left[p^{\beta_{1}+\beta_{2}}\right]} \cdots K_{\beta_{s}}^{\left[p^{\beta_{1}+\cdots+\beta_{s-1}}\right]} .
$$

Proposition 2.1. Fix any $e \geq 1$, and let $\mathcal{F}_{<e}$ be the $S$-subalgebra of $\mathcal{F}\left(E_{S}\right)$ generated by $\mathcal{F}^{0}\left(E_{S}\right), \ldots, \mathcal{F}^{e-1}\left(E_{S}\right)$. We have that $\mathcal{F}_{<e} \cap \mathcal{F}^{e}\left(E_{S}\right)=L_{e} f^{e}$.

Received by the editors June 5, 2009 and, in revised form, December 16, 2009.

2010 Mathematics Subject Classification. Primary 13A35, 13E10.

(c)2010 American Mathematical Society 2381 
Proof. Any element in $\mathcal{F}_{<e} \cap \mathcal{F}^{e}\left(E_{S}\right)$ can be written as a sum of elements of the form $\phi_{1} \cdots \phi_{s}$, where for all $1 \leq j \leq s$ we have $\phi_{j} \in \mathcal{F}^{\beta_{j}}\left(E_{S}\right)\left(1 \leq \beta_{j}<e\right)$ and $\beta_{1}+\cdots+\beta_{s}=e$. Each such $\phi_{j}$ equals $a_{j} f^{\beta_{j}}$ where $a_{j} \in K_{\beta_{j}}$, so

$$
\begin{aligned}
\phi_{1} \cdots \phi_{s} & =a_{1} f^{\beta_{1}} a_{2} f^{\beta_{2}} a_{3} f^{\beta_{3}} \cdots a_{s} f^{\beta_{s}} \\
& =a_{1} a_{2}^{p_{1}^{\beta}} a_{3}^{p^{\beta_{1}+\beta_{2}}} \cdots a_{s}^{p^{\beta_{1}+\cdots+\beta_{s-1}}} f^{\beta_{1}+\cdots+\beta_{s}} \in L_{e} f^{e},
\end{aligned}
$$

hence $\mathcal{F}_{<e} \cap \mathcal{F}^{e}\left(E_{S}\right) \subseteq L_{e} f^{e}$.

On the other hand, for all $1 \leq \beta_{1}, \ldots, \beta_{s}<e$ such that $\beta_{1}+\cdots+\beta_{s}=e$,

$$
K_{\beta_{1}} K_{\beta_{2}}^{\left[p^{\beta_{1}}\right]} K_{\beta_{3}}^{\left[p^{\beta_{1}+\beta_{2}}\right]} \cdots K_{\beta_{s}}^{\left[p^{\beta_{1}+\cdots+\beta_{s-1}}\right]} \subseteq\left(I^{\left[p^{\beta_{1}+\cdots+\beta_{s}}\right]}: I\right)=\left(I^{\left[p^{e}\right]}: I\right),
$$

so $L_{e} f^{e} \subseteq\left(I^{\left[p^{e}\right]}: I\right) f^{e}=\mathcal{F}^{e}\left(E_{S}\right)$. A similar argument to the one in the previous paragraph shows that we also have

$$
K_{\beta_{1}} K_{\beta_{2}}^{\left[p^{\beta_{1}}\right]} K_{\beta_{3}}^{\left[p^{\beta_{1}+\beta_{2}}\right]} \cdots K_{\beta_{s}}^{\left[p^{\left.\beta_{1}+\cdots+\beta_{s-1}\right]}\right.} f^{e} \subseteq \mathcal{F}_{<e},
$$

and we deduce that $L_{e} f^{e} \subseteq \mathcal{F}_{<e} \cap \mathcal{F}^{e}\left(E_{S}\right)$.

Now fix $I$ to be the ideal generated by $x y$ and $y z$. We show that $\mathcal{F}(M)$ is not a finitely generated $S$-algebra.

Proposition 2.2. For all $e \geq 1, K_{e}$ is generated by

$$
\left\{x^{p^{e}} y^{p^{e}-1}, x^{p^{e}-1} y^{p^{e}-1} z^{p^{e}-1}, y^{p^{e}-1} z^{p^{e}}\right\} .
$$

Proof. For any $q>1$,

$$
\begin{aligned}
\left(x^{q} y^{q}, y^{q} z^{q}\right):(x y, y z) & =\left(\left(x^{q} y^{q}, y^{q} z^{q}\right): x y\right) \cap\left(\left(x^{q} y^{q}, y^{q} z^{q}\right): y z\right) \\
& =\left(x^{q-1} y^{q-1}, y^{q-1} z^{q}\right) \cap\left(x^{q} y^{q-1}, y^{q-1} z^{q-1}\right) \\
& =\left(x^{q} y^{q-1}, x^{q-1} y^{q-1} z^{q-1}, x^{q} y^{q-1} z^{q}, y^{q-1} z^{q}\right) \\
& =\left(x^{q} y^{q-1}, x^{q-1} y^{q-1} z^{q-1}, y^{q-1} z^{q}\right) .
\end{aligned}
$$

Theorem 2.3. The $S$-algebra $\mathcal{F}\left(E_{S}\right)$ is not finitely generated.

Proof. It is enough to show that for all $e \geq 1, \mathcal{F}\left(E_{S}\right)$ is not in $\mathcal{F}_{<e}$, and we establish this by showing that the generator $x^{p^{e}} y^{p^{e}}-1$ of $K_{e}$ is not in $L_{e}$.

Since $L_{e}$ is a sum of monomial ideals, $x^{p^{e}} y^{p^{e}-1} \in L_{e}$ if and only if $x^{p^{e}} y^{p^{e}-1}$ is in one of the summands. So we now fix $e \geq 1$ and $1 \leq \beta_{1}, \ldots, \beta_{s}<e$ such that $\beta_{1}+\cdots+\beta_{s}=e$, and we show that the ideal

$$
K_{\beta_{1}} K_{\beta_{2}}^{\left[p^{\beta_{1}}\right]} K_{\beta_{3}}^{\left[p^{\beta_{1}+\beta_{2}}\right]} \cdots K_{\beta_{s}}^{\left[p^{\beta_{1}+\cdots+\beta_{s-1}}\right]}
$$

does not contain $x^{p^{e}} y^{p^{e}-1}$.

Since $z$ does not occur in $x^{p^{e}} y^{p^{e}-1}$, it is enough to show that with $J_{e}=x^{p^{e}} y^{p^{e}-1} R$,

$$
J_{\beta_{1}} J_{\beta_{2}}^{\left[p^{\beta_{1}}\right]} J_{\beta_{3}}^{\left[p^{\beta_{1}+\beta_{2}}\right]} \cdots J_{\beta_{s}}^{\left[p^{\beta_{1}+\cdots+\beta_{s-1}}\right]}
$$

does not contain $x^{p^{e}} y^{p^{e}-1}$. The exponent of $x$ in the generator of the product above is

$$
p^{\beta_{1}+\left(\beta_{1}+\beta_{2}\right)+\cdots+\left(\beta_{1}+\cdots+\beta_{s}\right)}>p^{\beta_{1}+\cdots+\beta_{s}}=p^{e},
$$

where the inequality follows from the fact that we must have $s>1$. 


\section{A COnjecture}

Although the example in section 2 settles the question raised in [LS], one might still raise the question of whether such examples exist over "nice" rings, e.g., normal domains.

Let $\mathbb{K}$ be a field of prime characteristic $p$, let $R=\mathbb{K} \llbracket x, y, z, u, v, w \rrbracket$ and let $I$ be the ideal generated by the $2 \times 2$ minors of the matrix $\left(\begin{array}{lll}x & y & z \\ u & v & w\end{array}\right)$. The ring $S=R / I$ is a normal, Cohen-Macaulay domain (cf. Theorem 7.3.1 in $[\mathrm{BH}]$ ). Let $E_{S}$ be the injective hull of the residue field of $S$ and, as before, for all $e \geq 1$ let $\mathcal{F}_{<e}$ be the $S$-subalgebra of $\mathcal{F}^{e}\left(E_{S}\right)$ generated by $\mathcal{F}^{1}\left(E_{S}\right), \ldots, \mathcal{F}^{e-1}\left(E_{S}\right)$. Note that $\mathcal{F}^{0}\left(E_{S}\right)=S$.

Conjecture 3.1. For all $e \geq 1, \mathcal{F}^{e}\left(E_{S}\right)$ is not contained in $\mathcal{F}_{<e}$, and hence $\mathcal{F}^{e}\left(E_{S}\right)$ is not a finitely generated $S$-algebra.

I have tested this conjecture using the computer system Macaulay 2 (GS), and, for example, in characteristic 2 , it holds for $1 \leq e \leq 6$.

\section{REFERENCES}

[BH] W. Bruns and J. Herzog. Cohen-Macaulay rings. Cambridge Studies in Advanced Mathematics, 39. Cambridge University Press, Cambridge, 1993. MR1251956 (95h:13020)

[GS] D. Grayson and M. Stillman. Macaulay 2 - a software system for algebraic geometry and commutative algebra, available at http://www.math.uiuc.edu/Macaulay2.

[K] M. Katzman. Parameter-test-ideals of Cohen-Macaulay rings. Compositio Mathematica, 144 (2008), pp. 933-948. MR2441251 (2009d:13030)

[LS] G. Lyubeznik and K. E. Smith. On the commutation of the test ideal with localization and completion. Transactions of the AMS 353 (2001), no. 8, pp. 3149-3180. MR1828602 (2002f:13010)

Department of Pure Mathematics, University of Sheffield, Hicks Building, Sheffield S3 7RH, United KingDom

E-mail address: M.Katzman@sheffield.ac.uk 